\title{
The tertiary gustatory center in sunfishes is not nucleus glomerulosus
}

\author{
Mario F. Wullimann \\ Neurobiology Unit, Scripps Institution of Oceanography and Department of Neurosciences, A-001, \\ University of California, San Diego, La Jolla, CA 92093 (U.S.A.)
}

(Received 23 September 1987; Revised version received 3 November 1987; Accepted I6 November 1987)

Key words: Diencephalon; Gustatory system; Nucleus glomerulosus; Percomorph teleost; Visual system

Injection of horseradish peroxidase into the secondary gustatory nucleus of the green sunfish, Lepomis cyanellus, resulted in retrogradely filled neurons bilaterally in the viscerosensory column of the brainstem and in anterograde transport revealing ipsilateral terminal fields in the preglomerular tertiary gustatory nucleus, the nucleus of the torus lateralis and the central and periventricular nucleus of the inferior lobe. Thus, the glomerular nucleus of percomorph teleosts is not a tertiary gustatory center. It is proposed that the term 'nucleus glomerulosus' be reserved for the nucleus involved with vision and that the preglomerular subdivision involved in gustation be termed 'nucleus gustatorius tertius'.

The preglomerular area in teleosts is a complex diencephalic region consisting of several subdivisions [1], some of which are shown in Fig. 1A. Many of these receive ascending sensory information and some are reciprocally connected with the telencephalon (see ref. 7 for a review). In percomorph teleosts, a conspicuous, concentrically laminated structure is embedded in the center of the preglomerular area. This structure (Fig. 1B) has been termed nucleus glomerulosus [7; nucleus rotundus of Ito and Kishida: 3, 4], and it is largely involved in visual circuitry [7-10]. Although a histologically comparable nucleus cannot be recognized in cypriniform teleosts, attempts have been made to identify a homologous structure within the preglomerular complex of the latter teleosts. For example, a 'nucleus glomerulosus' in the crucian carp, Carassius carassius, has been shown to receive a tertiary gustatory projection after lesioning of, or horseradish peroxidase (HRP) injections into, the secondary gustatory nucleus $[5,6]$. If nucleus glomerulosus of percomorphs is homologous to this tertiary gustatory center demonstrated in Carassius, then it would consequently be expected to receive a substantial projection from the secondary gustatory nucleus.

During an experimental connectional study of the valvula cerebelli in a percomorph, the green sunfish (Lepomis cyanellus), a fortuitous case resulted in an injec-

Correspondence: M.F. Wullimann, Neurobiology Unit, Scripps Institution of Oceanography and Department of Neurosciences, A-001, University of California, San Diego, La Jolla, CA 92093. U.S.A. 

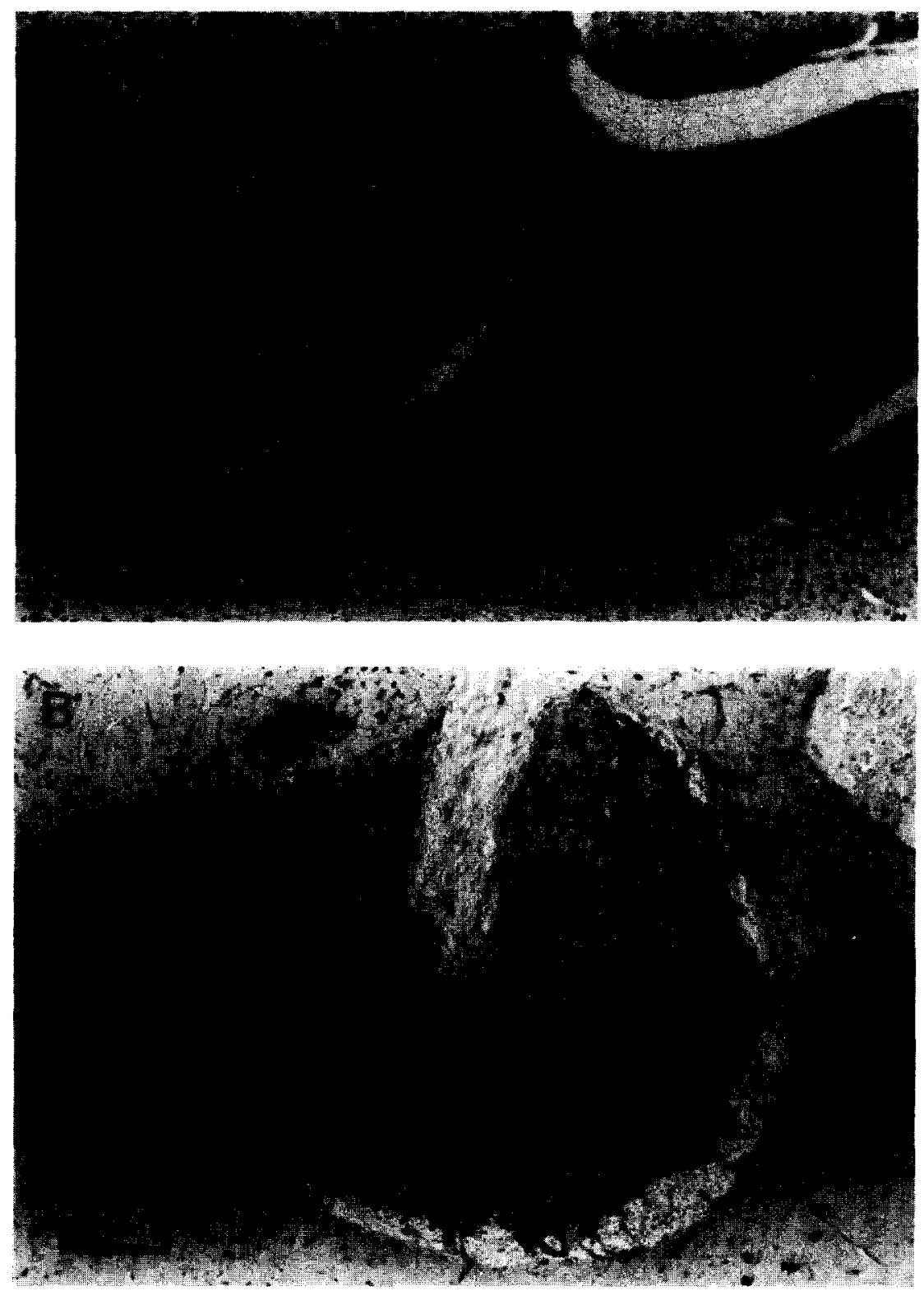

Fig. 1. Normal morphology of the preglomerular area in a Bodian-stained cross-section (A), and the glomerular nucleus in a Cresyl-stained cross-section (B) of Lepomis cyanellus. In A, the tertiary gustatory nucleus (NGT), as well as two additional tertiary gustatory centers, the nucleus of the torus lateralis (TLa) and the nucleus periventricularis recessus lateralis (NPLI) of the inferior lobe are shown. Arrow in B points to a single glomerulus within nucleus glomerulosus pars posterior (NGp). In this figure (as well as 3) medial is to the left, dorsal to the top. For abbreviations see Fig. 2. Bar $=0.1 \mathrm{~mm}$. 


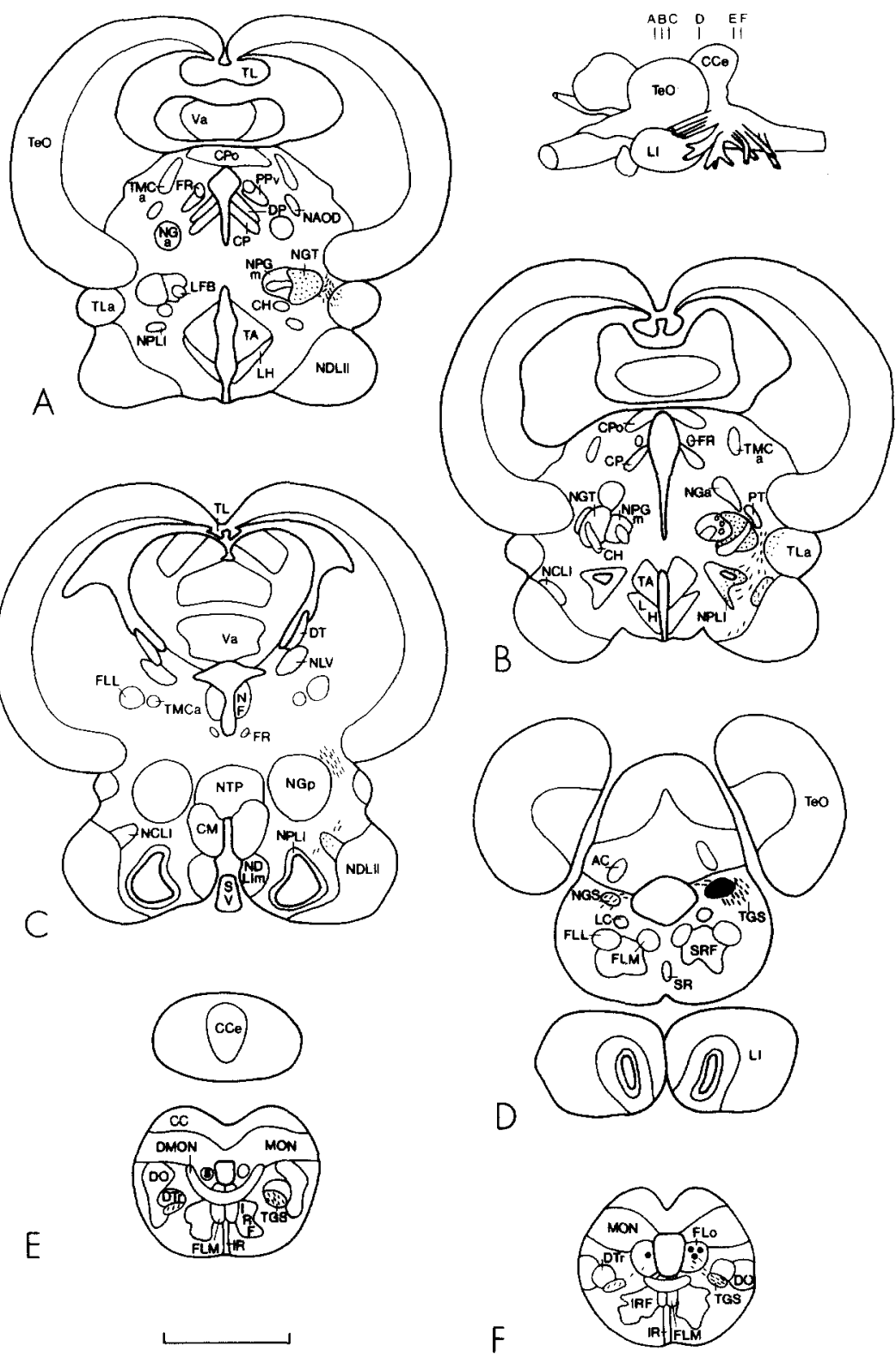

Fig. 2. Chartings of afferent and efferent connections of the secondary gustatory nucleus in Lepomis cyanellus. Black circles represent retrogradely filled cells, dots are terminals and dashes indicate the course of HRP-labeled fibers. A, nucleus anterioris thalami; AC, anterior cerebellar tract; $\mathrm{CC}$, crista cerebellaris; $\mathrm{CCe}$, corpus cerebelli; $\mathrm{CH}$, commissura horizontalis; $\mathrm{CM}$, corpus mammillare; $\mathrm{CP}$, nucleus centralis posterioris thalami; CPo, commissura posterior; DMON, decussation of the octavolateralis area; DO, nucleus octavus descendens; DP, nucleus dorsalis posterioris thalami; DT, nucleus tegmentalis dorsalis; DTr, descending trigeminal tract; FLL, fasciculus longitudinalis lateralis; FLM, fasciculus longitudinalis medialis; Flo, facial lobe; FR, fasciculus retroflexus; IR, inferior raphe; IRF, inferior reticular formation; LC, nucleus of the locus coeruleus; LFB, lateral forebrain bundle; LH nucleus lateralis hypothalami; LI, lobus inferior hypthalami; MON, nucleus octavolateralis medialis; NAOD, nucleus accessorius opticus dorsalis; 
tion of HRP (Sigma VI) into the secondary gustatory nucleus. In Lepomis, this nucleus is very small (Fig. 2D) compared to the large one in Carassius. The chances of repeating this localized injection are therefore remote. The surgical and histological procedures are described in detail elsewhere [11].

In Lepomis labeled fibers leave the secondary gustatory nucleus ipsilaterally and course rostrally (Fig. 2D). The fibers pass laterally to nucleus glomerulosus without entering it (Fig. 2C), and terminate heavily in one of the several more rostrally located subdivisions of the preglomerular area (Figs. 1A, 2A,B and 3), which lies immediately rostral to the posterior part of nucleus glomerulosus. This preglomerular division has been termed nucleus glomerulosus in Carassius carassius [5, 6], but some authors have questioned the implied homology to the percomorph nucleus glomerulosus by using quotation marks for 'glomerulosus' [1]. The present paper will refer to it as nucleus gustatorius tertius. Sparser terminal fields can be observed in the medial aspect of the nucleus of the lateral torus and the central and periventricu-

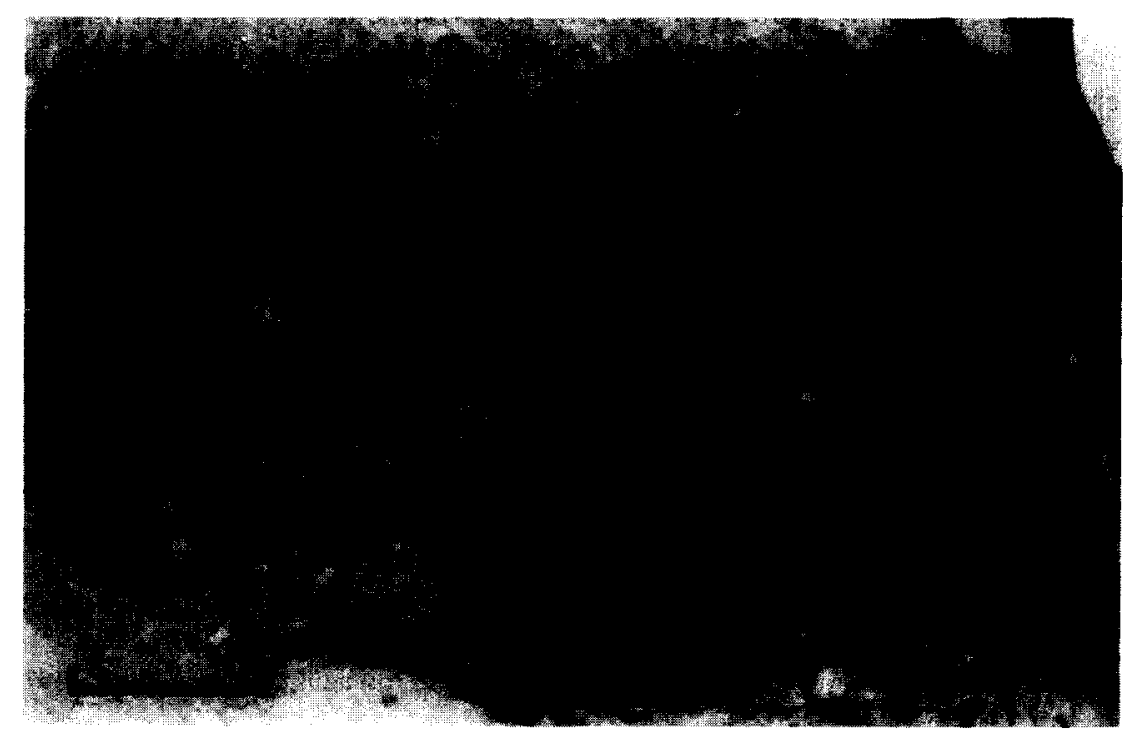

Fig. 3. Terminal field in the tertiary gustatory nucleus (NGT) visualized through anterograde HRP transport. NGT is surrounded by dashes, arrow points to a labeled fiber. Note that the more medial division of the preglomerular nucleus is not labeled. For abbreviations see Fig. 2 . Bar $=0.1 \mathrm{~mm}$.

NCLI, nucleus centralis of the inferior lobe; NDLII, nucleus diffusus of the inferior lobe, pars lateralis; NDLIm, nucleus diffusus of the inferior lobe, pars medialis; NF, nucleus of the fasciculus longitudinalis medialis; NGa, nucleus glomerulosus pars anterior; NGp, nucleus glomerulosus pars posterior; NGS, nucleus gustatorius secundarius; NGT, nucleus gustatorius tertius; NLV, nucleus lateralis valvulae; NPGm, nucleus preglomerulosus pars medialis; NPLI, nucleus periventricularis recessus lateralis of the inferior lobe; NTP, nucleus tuberis posterior; PPv, nucleus pretectalis periventricularis pars ventralis; PT, nucleus posterioris thalami; SR, superior raphe; SRF, superior reticular formation; s, sensory root of nervus facialis; SV, saccus vasculosus; TA, nucleus tuberis anterior; Tel, telencephalon; TeO, tectum opticum; TGS, tractus gustatorius secundarius; TL, torus longitudinalis; TLa, nucleus of the torus lateralis; TMCa, tractus mesencephalo-cerebellaris anterior; $\mathrm{Va}$, valvula cerebelli. $\mathrm{Bar}=1 \mathrm{~mm}$. 
lar nuclei of the inferior lobe (Fig. 2B,C). In addition, some labeled fibers can be traced to the lateral part of the diffuse nucleus of the inferior lobe.

Retrogradely filled neurons in the ipsilateral viscerosensory column of the brainstem (including primary sensory nuclei of the facial, glossopharyngeal and vagal nerves; Fig. 2F) project into the injected secondary gustatory nucleus within the ispilateral secondary gustatory tract (Fig. 2E). Some additional fibers originate from neurons in the contralateral viscerosensory column and cross within the commissure of the secondary gustatory nucleus to end in the injected nucleus. This is consistent with the finding of Morita et al. [5] that the ipsilateral facial lobe projects to both the ispi- and contralateral secondary gustatory nucleus.

The efferent projections of the secondary gustatory nucleus in Lepomis show essentially the same pattern of tertiary gustatory connections in percomorphs as has been demonstrated for cyprinids [5, 6] and silurids [2]. It is surprising, that while the secondary gustatory nucleus is much smaller in Lepomis than it is in cyprinids or silurids, the tertiary gustatory nucleus is of similar dimension.

Thus, in percomorphs a subdivision of the preglomerular area and not nucleus glomerulosus is a tertiary gustatory center. It is therefore suggested that the term nucleus glomerulosus be reserved for the highly differentiated and visually related nucleus in percomorphs [7] and that the nucleus termed 'nucleus glomerulosus' in cypriniforms be termed nucleus gustatorius tertius.

This work was supported by a grant from the Swiss National Science Foundation to the author and NIH grants to R.G. Northcutt. I thank R.G. Northcutt, R.L. Puzdrowski and G.F. Striedter for reading the manunscript and discussions on the larger topics related to this paper.

I Braford, M.R., Jr. and Northcutt, R.G., Organization of the diencephalon and pretectum in ray-finned fishes. In R.E. Davis and R.G. Northcutt (Eds.), Fish Neurobiology, Vol. 2, University of Michigan Press, Ann Arbor, 1983, pp. 117-163.

2 Finger, T.H., The gustatory system in teleost fish. In R.G. Northcutt and R.E. Davis (Eds.), Fish Neurobiology, Vol. 1, University of Michigan Press, Ann Arbor, 1983, pp. 285-309.

3 Ito, H. and Kishida, R., Organization of the teleostean nucleus rotundus, J. Morphol., 147 (1975) 89 107.

4 Ito, H. and Kishida, R., Synaptic organization of the nucleus rotundus in some teleosts, J. Morphol., 151 (1977) 397-417.

5 Morita, Y., Ito, H. and Masai, H., Central gustatory paths in the crucian carp, Carassius carassius, J. Comp. Neurol., 191 (1980) 119132.

6 Morita, Y., Murakami, T. and Ito, H., Cytoarchitecture and topographic projections of the gustatory centers in a teleost, Carassius carassius, J. Comp. Neurol., 218 (1983) 378-394.

7 Northcutt, R.G. and Wullimann, M.F., The visual system in teleost fishes: morphological patterns and trends. In J. Atema, R.R. Fay, A.N. Popper and W.N. Tavolga (Eds.), Sensory Biology of Aquatic Animals, Springer, New York, 1988, pp. 515-552.

8 Sakamoto, N. and Ito, H., Fiber connections of the corpus glomerulosum in an teleost, Navodon modestus, J. Comp. Neurol., 205 (1982) 291-298.

9 Striedter, G.F. and Northcutt, R.G., Connections of the superficial pretectum and nucleus isthmi in the sunfish. Lepomis cyanellus, Soc. Neurosci. Abstr., 12 (1986) 103.

10 Wullimann, M.F. and Northcutt, R.G., Vision in the coral reef: some visual circuits in Humu humus, Soc. Neurosci. Abstr., 12 (1986) 103.

11 Wullimann. M.F. and Northcutt. R.G., Connections of the valvula cerebelli in the green sunfish and in the common goldfish, in preparation. 